\section{The British Thoracic Society guideline on pulmonary rehabilitation in adults: your opinion is noted}

We thank Professors McConnell and Gosselink for their interest in the British Thoracic Society (BTS) pulmonary rehabilitation guideline. ${ }^{12}$ We welcome the opportunity to highlight the robust, unbiased approach that the guideline development group (GDG) has followed in making their recommendations which have been accredited by National Institute of Health and Care Excellence. This process, according to the BTS production manual, is described in the guideline methodology. ${ }^{2}$ It included initial training, each article being considered by at least two GDG members, independent peer review at multiple stages and consultation periods for the public and stakeholders. Continuously updated declaration of interest forms were submitted.

The correspondents question why inspiratory muscle training (IMT) is not recommended as a routine adjunct to pulmonary rehabilitation. The guideline discusses the background to IMT and the nature of the trials undertaken, including a considered judgement of the risk of bias therein. The randomised controlled trials (RCTs) at lowest risk of bias found IMT was of no benefit as an adjunct to the primary aims of rehabilitation, and hence it was not routinely recommended. A similar statement has been adopted in the recent American Thoracic Society/ European Respiratory Society statement on pulmonary rehabilitation. ${ }^{3}$ The label of ' $1+$ ' is a standard assessment of the evidence level informing the recommendation, not of the strength of support for using the named intervention. As the trials included exercise rather than multidisciplinary pulmonary rehabilitation, the recommendation was at Grade B. A high-quality RCT of high-intensity IMT in parallel to pulmonary rehabilitation focusing on functional exercise capacity and health status is required. As we state, consideration of individual interventions such as role of oxygen or IMT in isolation was beyond the remit of the guideline. ${ }^{2}$

In relation to limb resistance training, the correspondents have omitted the first evidence statement documented in the guideline against this adjunct:

Resistance training in combination with aerobic training leads to greater improvements in peripheral muscle strength than aerobic training alone. (Evidence level 1+)

The recommendation that limb resistance training is used in addition to aerobic training to improve muscle strength follows from this and has been contextualised in this manner and approved by subsequent reviewers. The recommendation is extrapolated from exercise-based RCTs with a low risk of bias (ie, Grade B). ${ }^{2}$

In the relevant sections, reference to the importance of peripheral muscle strength regarding mortality and healthcare utilisation is made, including one publication by Professor Gosselink. The guideline correctly states that other benefits are beyond the scope of the document.

We hope that high-quality, unbiased research in pulmonary rehabilitation continues to accrue and thus guide future iterations of the BTS pulmonary rehabilitation guideline.

Charlotte E Bolton, ${ }^{1}$ John D Blakey, ${ }^{2}$ Mike D Morgan, ${ }^{3}$ on behalf of the BTS Pulmonary Rehabilitation Guideline Development Group for the Standards of Care Committee for the BTS

${ }^{1}$ Nottingham Respiratory Research Unit, School of Medicine, University of Nottingham, Nottingham, UK ${ }^{2}$ Clinical Sciences, Liverpool School of Tropical Medicine, Liverpool, UK

${ }^{3}$ Respiratory Medicine, Glenfield Hospital, Leicester, UK

Correspondence to Dr Charlotte Bolton, Nottingham Respiratory Research Unit, The University of Nottingham, Clinical Sciences Building, City Hospital Campus, Hucknall Road, Nottingham NG5 1PB, UK; charlotte.bolton@nottingham.ac.uk

Contributors CEB, JDB and MDM contributed to the writing of the letter and approved the final version.

Competing interests None.

Provenance and peer review Not commissioned; internally peer reviewed.

To cite Bolton CE, Blakey JD, Morgan MD, et al. Thorax 2014;69:388-389.

Received 25 October 2013

Accepted 26 October 2013

Published Online First 15 January 2014

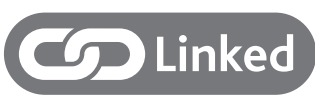

http://dx.doi.org/10.1136/thoraxjnl-2013-204684

Thorax 2014;69:388-389.

doi:10.1136/thoraxjnl-2013-204754

\section{REFERENCES}

1 McConnell AK, Gosselink R. British Thoracic Society guideline on pulmonary rehabilitation in adults: does objectivity have a sliding scale? Thorax 2014;69:387-8. 
2 Bolton CE, Bevan-Smith EF, Blakey JD, et al. British Thoracic Society Pulmonary Rehabilitation Guideline Development Group. The British Thoracic Society guideline on pulmonary rehabilitation in adults: accredited by NICE. Thorax 2013;68(Suppl 2): ii1-30.

3 Spruit MA, Singh SJ, Garvey C, et al. ATS/ERS Task Force on Pulmonary Rehabilitation. An official
American Thoracic Society/European Respiratory Society statement: key concepts and advances in pulmonary rehabilitation. Am J Respir Crit Care Med 2013;188:e13-64. 